\title{
A cellular automaton for modeling non-trivial biomembrane ruptures
}

\author{
Abhay Gupta ${ }^{1}$, Irep Gözen ${ }^{2,3,4}$, Michael Taylor ${ }^{1 *}$ \\ ${ }^{1}$ Department of Mechanical Engineering, Santa Clara University, Santa Clara, California, USA \\ ${ }^{2}$ Centre for Molecular Medicine Norway, Faculty of Medicine, University of Oslo, 0318 Oslo, Norway \\ ${ }^{3}$ Department of Chemistry, Faculty of Mathematics and Natural Sciences, University of Oslo, 0315 Oslo, Norway \\ ${ }^{4}$ Department of Chemistry and Chemical Engineering, Chalmers University of Technology, SE-412 96 Göteborg, Sweden
}

\begin{abstract}
A novel cellular automaton (CA) for simulating biological membrane rupture is proposed. Constructed via simple rules governing deformation, tension, and fracture, the CA incorporates ideas from standard percolation models and bond-based fracture methods. The model is demonstrated by comparing simulations with experimental results of a double bilayer lipid membrane expanding on a solid substrate. Results indicate that the CA can capture non-trivial rupture morphologies such as floral patterns and the saltatory dynamics of fractal avalanches observed in experiments. Moreover, the CA provides insight into the poorly understood role of inter-layer adhesion, supporting the hypothesis that the density of adhesion sites governs rupture morphology.
\end{abstract}

\section{Introduction}

Under mechanical stress, biological membranes have been shown to display non-trivial pore morphologies and dynamics ${ }^{1}$ in addition to the previously known circular transient pores. The peculiar morphologies comprise large flower-like shapes growing continuously referred to as 'floral' pores, and fractures appearing intermittently in 'fractal' patterns. Both rupture types occur in the exact same experimental conditions with the identical membrane compositions, solid substrates and ambient buffers. The factors which influence the fate of the rupture mode are not exactly understood and the detailed mechanisms remain to be elucidated.

We hypothesize that the density of adhesion sites of the membrane to the underlying layer, defines the rupture type. In our experiments, where a double lipid bilayer membrane (DLBM) is formed on a solid substrate via self-spreading of a lipid reservoir, the adhesion is most likely a result of pinning between the two bilayers mediated by $\mathrm{Ca}^{2+1,2}$. In a recently reported experimental system where fractal pores in solidsupported membranes were observed, the adhesion was created via biotin-avidin bridging of the two bilayers ${ }^{3}$. The water layer between the two bilayers in a DLBM stack through which the $\mathrm{Ca}^{2+}$-mediated pinning is established, is maximum a few tens of nanometers thick. An intact DLBM with a diameter of $100 \mu \mathrm{m}$ and an inter-bilayer space of $10 \mathrm{~nm}$ has a volume of about $750 \mathrm{fL}^{4}$. This makes the direct observations, for instance the visualization of the labeled $\mathrm{Ca}^{2+}$ ions, challenging. Therefore, we utilize computer modelling, which allows us to independently tune the potentially influential parameters affecting the rupture morphology to understand both their individual impact and, consequentially, the detailed mechanisms involved.

Researchers have noted similarity between the floral and fractal lipid rupture morphologies and the interaction of immiscible fluids in porous media ${ }^{1,4}$. In particular, lipid fracture is related to viscous fingering and percolation phenomena. These types of instabilities have been of interest to researchers investigating oil recovery ${ }^{5}$, fluid mixing ${ }^{6}$, soil physics ${ }^{7}$, and biological tissue and organ engineering ${ }^{8,9}$. 
One of the most well-established computational paradigms for exploring percolation and growth phenomena is spatial simulation, of which cellular automata (CA) are a prominent example ${ }^{10,11}$. CA trace their roots back to von Neumann's desire to create self-replicating machines ${ }^{12,13}$ and were popularized by Conway's discovery of the Game of Life ${ }^{14}$. Subsequent academic interest in CA owes much to the work of Wolfram ${ }^{15}$. A cellular automaton consists of a grid of cells (commonly in one or two dimensions) that are assigned a specific state (or states). In the simplest of CA, this state is binary, 0 or 1 , on or off, alive or dead, etc.; although, this is not a necessity. These states evolve through the application of a few very simple rules, e.g. "randomly select a 0 neighbor of a 1 cell and make it 1." Despite their simplicity, CA are able to capture a range of complex behavior, even emergent behavior, in the areas of growth, aggregation, segregation, and percolation ${ }^{10,11}$.

While CA are relatively simple, numerical methods for modeling fracture typically are not. This is due to the fact that ruptures involve discontinuities that hamper discretizations based on spatial derivatives. Peridynamics is a continuum mechanics theory that avoids this problem by being based on an integral equation of motion ${ }^{16,17}$. In its mesh-free discretization, it models particles interacting via bonds, which can break causing fracture ${ }^{18}$. Our recently published mesh-free peridynamic model of biomembrane ruptures revealed that the fluid biological membranes favoring fractal morphologies could adopt a nonzero shear modulus ${ }^{19}$. While the model captures circular and floral patterns and their associated dynamics, it does not capture the saltatory dynamics of fractal rupture nor the very fine fractal patterns observed experimentally. This is likely due to two primary causes. First, we used a very simple linear fluid model for the lipid bilayer. Adapting a more appropriate constitutive equation, e.g. a Helfrich model, to the peridynamic theory would lead to more accurate results. In addition, our peridynamic approach utilized mass scaling to increase the stable time-step size and reduce simulation times. This may have negatively impacted the accuracy of the rupture dynamics. Even with GPU parallelization and mass scaling, a peridynamic simulation of the aforementioned experimental system takes on the order of tens of minutes.

In this work, we propose a new innovative CA incorporating ideas from both standard percolation models as well as our previous peridynamics model that captures circular, floral, and fractal avalanche morphology and their associated dynamics in single framework. In contrast to our previous peridynamic model, a single CA simulation runs on the order of minutes serially on a typical desktop computer. We illustrate the CA model through simulations of an expanding DLBM on a solid substrate and compare with experimental results. The goal of this new model is to determine the rules underlying pattern formation and saltatory dynamics of lipid membrane rupture in order to gain insight on the role of pinning in this process, which is still poorly understood. This is important because a better understanding of pore formation in biological membranes can help in finding possible mechanisms behind cell-integrity related disease ${ }^{20-22}$ and also would help establish a ground for improvement of related medical treatment methods, e.g., drug delivery ${ }^{23}$, gene therapy ${ }^{24}$.

\section{Materials and Methods}

\section{Preparation of lipid suspension:}

Lipids and lipid fluorophore: Soybean Polar Lipid Extract(SPE); E. coli Polar Lipid Extract(ECPE); 1-oleoyl-2(6-((4,4-difluoro-1,3-dimethyl-5-(4-methoxyphenyl)-4-bora-3a,4a-diaza-s-indacene-2-

propionyl)amino)hexanoyl)-sn-glycero-3- phosphoethanolamine (TopFluor ${ }^{\mathrm{TM}} \mathrm{TMR} P \mathrm{PE}$ ), were obtained from Avanti Polar Lipids (AL, USA).

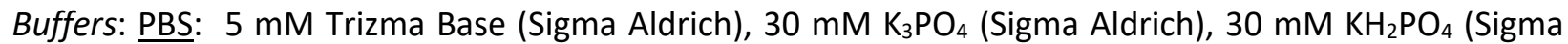


Aldrich), $3 \mathrm{mM} \mathrm{MgSO}{ }_{4} \cdot 7 \mathrm{H} 2 \mathrm{O}$ (Sigma Aldrich) and $0.5 \mathrm{mM} \mathrm{Na}_{2}$ EDTA (Sigma Aldrich). The pH was adjusted to 7.4 with $\mathrm{H}_{3} \mathrm{PO}_{4}$. $\underline{\text { HEPES without } \mathrm{CaCl}_{2}}$ (for rehydration): $10 \mathrm{mM} \mathrm{HEPES} \mathrm{(Sigma} \mathrm{Aldrich),} 100 \mathrm{mM} \mathrm{NaCl}$ (Sigma Aldrich), the $\mathrm{pH}$ was adjusted to 7.8 with $\mathrm{NaOH}$. HEPES with $\mathrm{CaCl}_{2}$ (ambient buffer for spreading): $10 \mathrm{mM}$ HEPES (Sigma Aldrich), $100 \mathrm{mM} \mathrm{NaCl}$ (Sigma Aldrich), 2,5 mM or $4 \mathrm{mM} \mathrm{CaCl}_{2}$ (Sigma Aldrich). The $\mathrm{pH}$ was adjusted to 7.8 with $\mathrm{NaOH}$. Above $4 \mathrm{mM} \mathrm{Ca}^{2+}$, the spreading and rupturing occurs too rapid, making microscopy observations difficult. Below $0.8 \mathrm{mM} \mathrm{Ca}^{2+}$ spreading does not occur ${ }^{25}$. For the concentrations in between we have not yet been able to determine a direct relation between the concentration of $\mathrm{Ca}^{2+}$ and the density of the pinning points. Direct experimental observation is not straight-forward.

Lipids and lipid-conjugated fluorophore, all dissolved in chloroform, were mixed in the following ratios: SPE 50 wt \%, ECPE 49 wt \%, TopFluor TMR PE $1 \mathrm{wt} \%$. The volumes corresponding to the specified mass fractions of lipids, were transferred into a round-bottom flask leading in total to $3000 \mu \mathrm{g}$ of lipids in 300 $\mu \mathrm{l}$ chloroform $(10 \mathrm{mg} / \mathrm{ml})$. The solvent was evaporated in a rotary evaporator at $-80 \mathrm{kPa}$ for 6 hours, to form a dry lipid cake at the bottom of the flask. $3 \mathrm{ml}$ of PBS buffer and $30 \mu \mathrm{l}$ of glycerol were added to the flask and the mixture was placed at $+4^{\circ} \mathrm{C}$ overnight for swelling. The next day the flask was placed in an ultrasonic bath sonicator (VWR) at $30{ }^{\circ} \mathrm{C}$ for $15-30 \mathrm{~s}$, to form a lipid suspension containing giant multiand unilamellar vesicles.

\section{Surface fabrication:}

$\mathrm{SiO}_{2}$ coatings were produced in the clean room facility MC2 at Chalmers University of Technology, Sweden using MS 150 Sputter system (FHR Anlagenbau $\mathrm{GmbH}$ ). All depositions were applied on glass cover slips (\#1, Menzel-Gläser) which were pre-cleaned with oxygen plasma $2 \mathrm{~min}$ at $50 \mathrm{~W}$ using Plasma Therm BatchTop PE/RIE m/95. The final film thickness of $\mathrm{SiO}_{2}$ was, for fractal: $10 \mathrm{~nm}$, for mixed patterns: $84 \mathrm{~nm}$ and for floral pore formation: $15 \mathrm{~nm}$.

\section{Spreading:}

$4 \mu \mathrm{l}$ of the stock lipid suspension described above, was placed on a clean glass cover slip and desiccated for 20 minutes. The resulting dry film was rehydrated with HEPES buffer without $\mathrm{CaCl}_{2}$ for 3 minutes.

The re-hydrated sample containing lipid vesicles were placed on top of a $\mathrm{SiO}_{2}$ coated glass slides, in HEPES buffer with $\mathrm{Ca}^{2+}$ which leads to spreading of the multilamellar lipid vesicles (reservoirs) in form of a double lipid bilayer membrane (Fig. 1).

\section{Microscopy:}

A laser scanning confocal microscope (Leica SPX8, Germany) was used to visualize the experiments. A white light laser source was used for excitation of TopFluor ${ }^{\mathrm{TM}}$ TMR PE at $544 \mathrm{~nm}$ and emission was collected at $560-600 \mathrm{~nm}$ by a photomultiplier tube detector. All fluorescence micrographs were digitally enhanced and false colored.

\section{Numerical Model:}

Like the authors' previous peridynamic study ${ }^{19}$, we consider only the expanding and rupturing distal lipid bilayer in creating a two-dimensional model of the system. Accordingly, our CA are contained within a $n \times n$ square region of cells. Initially, all cells within a radius $R_{i}$ are considered to be part of the lipid bilayer. In each generation, the radius of the membrane is increased by one cell, which continues until a

final radius $R_{f}=n / 2$ is reached, whereupon the simulation ends. Both $n$ and $R_{i}$ are user-specified parameters.

In addition to a Boolean state governing whether or not a cell is within the lipid membrane, we assign states describing whether or not a cell is a pinning site (Boolean), its tension (floating point number), and whether or not it is ruptured (Boolean). Pinning location and behavior are initialized stochastically, while tension and rupture are governed by fixed stiffness and critical tension parameters, respectively, which 
are assigned material properties. We describe pinning, tension, and fracture in more detail in the following sections. All simulations were performed using a specially-written program in Matlab 2015b (MathWorks), available in the electronic Supporting Information (SI), run on a desktop iMac computer.

\section{Pinning}

In the real physical system, pinning between distal and proximal layers is widespread with locations that are unpredictable. To account for this variability in our numerical model, we assign two pseudorandom numbers $\theta_{1}, \theta_{2} \in[0,1]$ to all cells within the lipid. Cells with $\theta_{1}$ less than a user-defined pinning probability $P_{\text {pin }}$ become a (dilute) pinning site. If, in addition, a pinned cell with $\theta_{2}$ less than a user-defined cluster probability $P_{\text {cluster }}$ become the root of a (dense) pinning cluster.

To create the cluster, we use a site percolation model ${ }^{10}$. First, cells in Moore's neighborhood (i.e., the eight adjacent cells) of the cluster root become a pin with a probability $P_{\text {perc }}$ based on their $\theta_{1}$ value. Next, cells in Moore's neighborhood of these new pins also become a pin with a probability $P_{\text {perc }}$ based on their $\theta_{1}$ value. This process is continued recursively until there are no more neighbors with $\theta_{1}<P_{\text {perc }}$, at which point the cluster has reached its terminal size. Fig. 1 shows the schematic representation of the hypothesized dilute and dense pinning as well as a typical CA snapshot with pinning sites in both dilute and dense cases present.

We note that our CA is deterministic. While the system is initialized pseudorandomly, the values of $\theta_{1}$ and $\theta_{2}$ as well as the underlying rules remain fixed throughout the simulation.

\section{Tension}

As the distal lipid layer expands, its tension increases. The presence of pinning sites acts as local tension raisers. Accordingly, all pinned cells in our model are assigned a tension $T$ comprising both the spreading tension $T_{s}$ and a pinning tension $T_{p}$. In the physical system, the spreading is driven by a gradient between the surface adhesion tension and the internal tension of the multilamellar reservoir (i.e., Marangoni flow). Approximating the membrane as incompressible, the spreading tension at points located distance $r$ from the membrane center is ${ }^{4}$

$T_{s}=\left(\frac{T_{\text {adhesion }}-T_{\text {reservoir }}}{R_{\text {curr }}}\right) r+T_{\text {reservoir }}$

where $T_{\text {adhesion }}$ is the tension due to the lipid's adhesion to the surface, $T_{\text {reservoir }}$ is the tension of the multilamellar reservoir, and $R_{\text {curr }}$ is the current radius of the lipid membrane. For an expanding membrane, $T_{\text {adhesion }}>T_{\text {reservoir }}$.

The pinning tension arises from the expanding membrane pulling on the fixed pinned cells. We assume a linear model for the pinning tension governed by a stiffness parameter $c$

$T_{p}=\frac{c\left(R_{c u r r}-r\right)}{R_{f}}$,

which is higher for those pinned cells closer to the center of the membrane. This is in contrast to the spreading tension, which is higher for those cells nearest outer edge of the membrane. In our model, 
we choose $T_{\text {adhesion }}, T_{\text {reservoir }}$, and $c$ such that the total tension $T=T_{s}+T_{p}$ is highest at the center of the membrane (i.e., the pinning tension dominates) as that gives the best agreement with experiments. Figure 2a shows typical tension profiles for several $R_{\text {curr }}$ values in a CA with $R_{f}=100$.

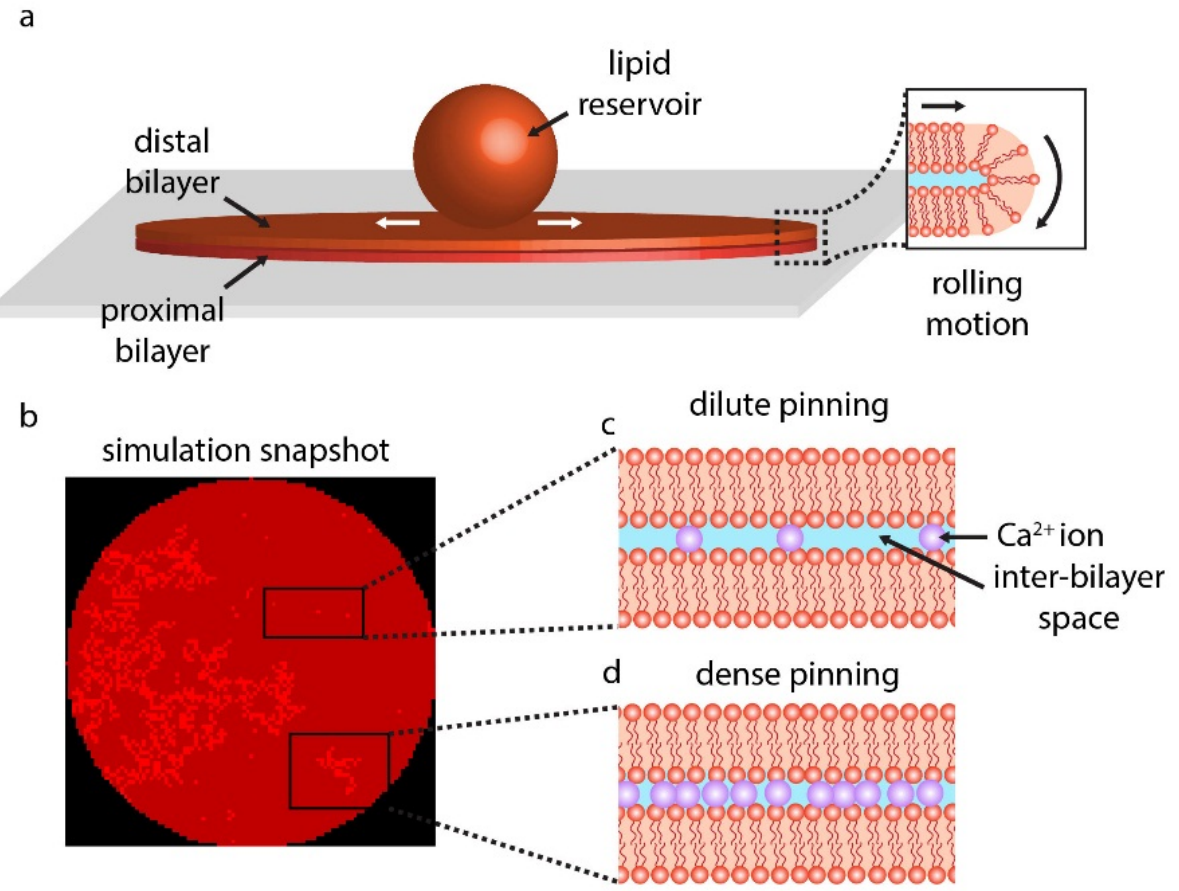

Figure 1 | Overview of the experiment and the model. (a) The experiment starts with placing a multilamellar reservoir on a $\mathrm{SiO}_{2}$ substrate which leads to spreading of the reservoir as a circular double lipid bilayer membrane (DLBM). The peripheries of the circular membrane are depicted open to ease the understanding of the reader. In reality the edges are closed (inset) to avoid the exposure of the hydrophobic moieties of the phospholipid molecules to the aqueous buffer. (b) A simulation snapshot showing pinning points (bright red regions). The model is investigating the hypothesis that the type of rupture morphology and associated rupture dynamics, are determined by the density and distribution of pinning. The pinning occurs due to the $\mathrm{Ca}^{2+}$ bridging the two bilayers. (c) The dilute pinning, relatively larger space in between the pinning sites, cause large pores or floral patterns. (d) The dense pinning, where $\mathrm{Ca}^{2+}$ ions are packed closely, lead to fractal ruptures. In the simulations, the dilute pinning is programmed by randomly assigning cells within the lipid area as pinned; and dense pinning, by randomly placing pinned clusters based on a site percolation algorithm.

\section{Fracture}

In every CA generation, each pinned cell's tension $T$ is checked against a critical tension $T_{\text {crit }}$. If the tension exceeds the critical tension, the cell is fractured. This straightforward type of fracture criteria is common among bond-based mechanics models, of which our prior peridynamic work is an example ${ }^{19}$. The growth and spread of the rupture are determined by the type of pin the cell is (Fig. 2b-g). For dilute pins (Fig. 2bd), fracture is an increasing circular pore, the radius of which grows by one cell in each CA generation. Cells within this radius are fractured regardless of their pinning state. If a pore of radius $R_{\text {pore }}$ reaches another pinned cell that hasn't fractured, it is immediately fractured (regardless of tension). Moreover, all cells within a radius of $R_{\text {pore }}$ of the newly fractured cell are fractured as well. This process of pore 
growth possibly initiating new pores is done recursively within a given CA generation and models the continuous linear increase in floral pore area observed experimentally ${ }^{1}$.

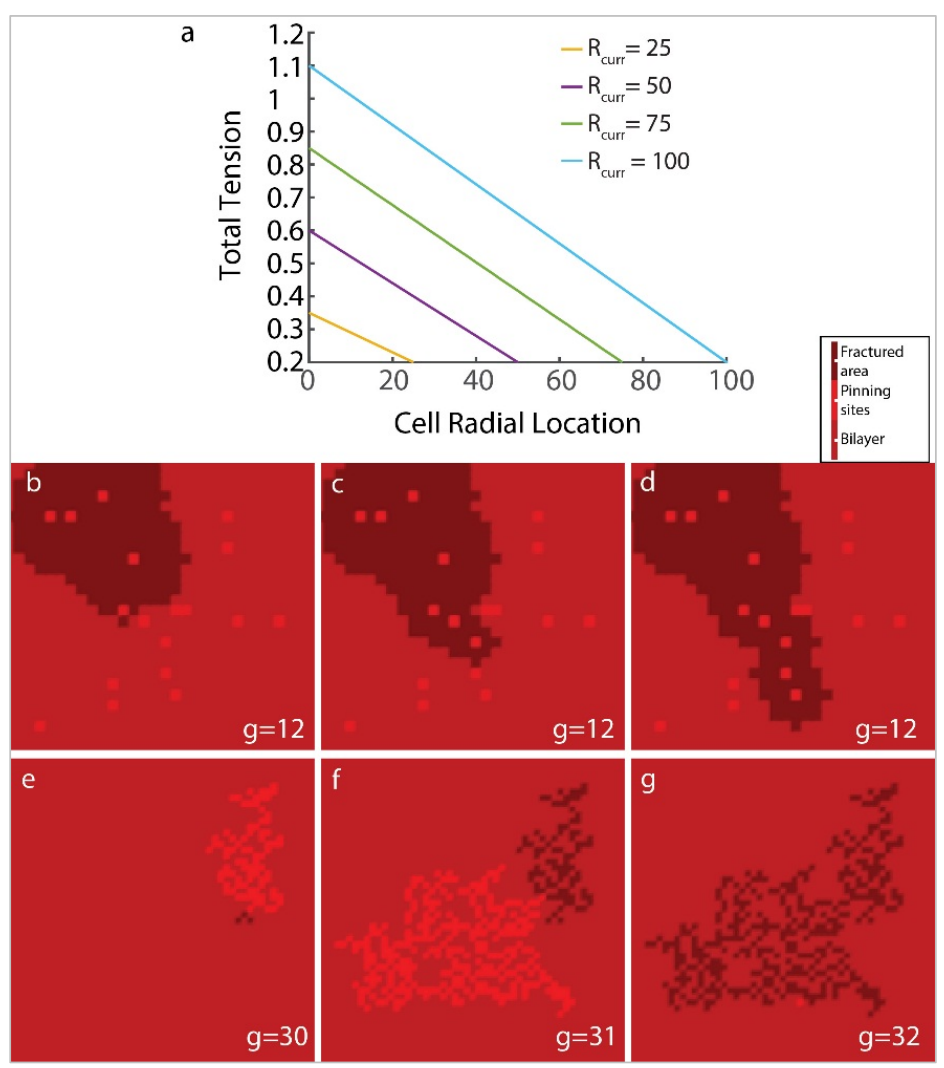

Figure 2 | Details of pore formation in the CA model. (a) The total tension $T=T_{s}+T_{p}$ as a function of radius $r$ is shown for various $R_{\text {curr }}$ and a final radius $R_{f}=100$ when the $C T P=0$. (b-d) Typical fracture event for dilute pins. When a floral pore reaches an unbroken pin (b), a new circular pore forms at that pin and grows radially outward. The new pore contacts neighboring unbroken pins, causing new pores to form (c), which grow and cause other neighboring pins to rupture (d). (e-g) Typical fracture event for cluster pins. A small broken cluster begets an offshoot cluster of pins (e), which ruptures under higher tension in a succeeding generation, begetting another offshoot cluster (f), which ruptures in turn during a succeeding generation (g).

For pinned clusters (Fig. 2e-g), rupture growth proceeds differently. Once the critical tension is exceeded by a single cell in a cluster, all cells in the cluster (and any cluster pin cells in adjoining clusters) are fractured simultaneously. Rather than releasing energy by creating a growing circular pore, fractured clusters create a chain of many small static pores. In addition, they generate a new unbroken offshoot cluster with an offshoot probability $P_{\text {offshoot }}$. This is a realistic assumption since it is anticipated that in the experiments when a pore opens, $\mathrm{Ca}^{2+}$ ions in the ambient buffer rapidly penetrates into the interbilayer space, dynamically inducing further pinning. This offshoot cluster is rooted the neighboring cell with the lowest $\theta_{1}$ such that $\theta_{1}<P_{\text {offshoot }}$ and created following the same site percolation process described earlier. If there is no such cell meeting this criterion, no offshoot cluster is formed. The offshoot cluster is initialized with a reduced tension 
$T_{r}=A P *\left(T_{\text {crit }}-T_{\text {break }}\right)$,

where $T_{\text {break }}$ was the tension that caused the initiating cluster to break and $A P$ is a positive avalanche parameter. The reduced tension accounts for the energy released by the fracture of the initiating cluster and subsequent relaxing of the membrane. $A P$ governs the saltatory behavior of fractal rupture observed in experiments ${ }^{1,3}$. When $A P=0$, saltatory growth is suppressed. As $A P$ is increased, the delay between cluster ruptures becomes more pronounced. A more detailed analysis of this behavior is given in the Results and Discussion section.

a

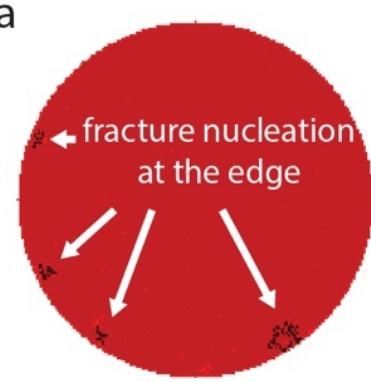

C

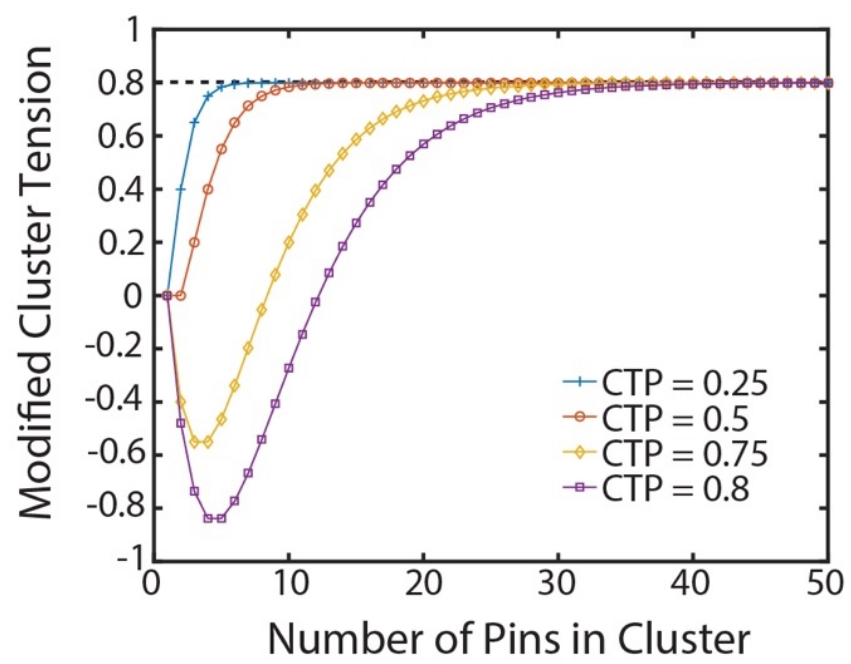

b

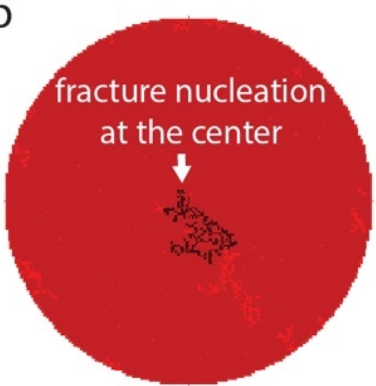


$T_{\text {cluster }}=T_{\text {crit }}\left(1-N_{\text {cluster }} * C T P^{N_{\text {cluster }}}\right)$

and $\operatorname{CTP} \in[0,1]$ is a cluster tension parameter used to differentiate the behavior of small clusters (i.e., those for which $N_{\text {cluster }}<20$ ) versus larger clusters. For CTP $>0.5, T_{\text {cluster }}$ increases the tension on clusters of all sizes up to a maximum of $T_{\text {crit }}$; although, its effect is larger on large clusters. In this case, ruptures will tend to start at the outer edge (Fig. 3a). On the other hand, for CTP $<0.5, T_{\text {cluster }}$ reduces the tension on small clusters while increasing the tension of larger clusters. In this case, ruptures tend to initiate towards the center of the lipid (Fig. 3b). Figure $3 c$ shows $T_{\text {cluster }}$ as a function of cluster size for a range of $C T P$ values.

\section{Results and Discussion}

We start the experiments by placing a lipid reservoir, a multilamellar phospholipid vesicle (MLV), on a $\mathrm{SiO}_{2}$ surface. Upon contact with the surface, the MLV spontaneously starts to wet the surface as a circular double lipid bilayer membrane (DLBM) (Fig. 1a) ${ }^{1,26}$. The periphery of the circular membrane in Figure 1a is depicted open, to facilitate the understanding of the reader. In reality the DBLM is intact, and the edges are closed (inset) to avoid the exposure of the hydrophobic moieties of the phospholipid molecules to the aqueous buffer. The continuous spreading increases the tension of the membrane and when exceeds the lysis tension $\left(5-10 \mathrm{mN} \mathrm{m}^{-1}\right)$, the membrane ruptures ${ }^{1}$. The morphology and dynamics of the biomembrane ruptures mainly follow two forms: large floral pores which continuously progress; and fine, fractal pores which occur intermittently, with waiting times in between ${ }^{19,1}$. Occasionally, we observe both ruptures on the same membrane patch. In this model, we hypothesize that the number and distribution of the pinning sites, most possibly caused by the $\mathrm{Ca}^{2+}$ ion bridging the two bilayers, determine the morphology of the rupture (Figure 1b-d). Since lipid membranes are known to intrinsically contain defects $^{27,28}$, allowing the penetration of ions through the membranes, and since the micrometer-sized pores forming in our experiments would promote further $\mathrm{Ca}^{2+}$ binding between the two bilayers, we conclude that $\mathrm{Ca}^{2+}$-mediated inter-bilayer binding is most likely responsible for the pinning. An alternative means of pinning could be originating from surface defects ${ }^{29,} 30$ which would need to extend through nanoscale pores in the proximal (lower) bilayer and pin directly to the distal (upper) bilayer. The rupturing bilayer that is in the focus of our experiments however, is residing on the surface adhered bilayer and not directly on the solid substrate. This is an important distinction, as the lipid bilayers directly adhered onto a high energy substrate possesses different dynamics, do irreversibly strongly fuse to the surface and do not exhibit rupturing ${ }^{1}$. Rupturing only occurs if the bilayer moderately adheres to the layer underneath, and is sufficiently fluid to build up tension gradients. This is the case for the distal bilayer which can slide on the thin water layer $(10 \mathrm{~nm})$ residing on the proximal bilayer, i.e., in between the two stacked bilayers.

Figure $1 \mathrm{~b}$ shows a snapshot from our simulations, where pinning sites both individual and in clusters, have been randomly positioned throughout the circular patch (bright red). The singular pinning points represent individual or very few $\mathrm{Ca}^{2+}$ ions (Fig. 1c); and the clusters represent dense regions of $\mathrm{Ca}^{2+}$ bridging between the two layers (Fig. 1d).

Figure 4 shows membranes on a solid substrate and the corresponding CA simulations displaying the two distinct rupture morphologies, floral and fractal, as well as the combination of both (SI Movies). The membrane in the experiments is doped with a fluorescence dye, which makes it visible. The micrographs are taken from top view. In the experiments when a pore opens, the proximal membrane becomes visible 
through the opening in the distal membrane, represented by half the light intensity ${ }^{1}$. The lipid material which initially resides in the area before the pore opens, now migrates toward the edges of the lipid patch. In the simulations, different colors represent from the most to less intense red: the pinning sites, the bilayer (distal membrane), fractured area (the proximal membrane). The background (surface) is depicted as black.

The key parameters used in the CA simulations are given in Table 1. These were all chosen based on parameter studies to best match the experimental data and guided by the physical considerations described in the Numerical Model section. Specifically, we tuned the parameters to yield not only good qualitative agreement with the observed rupture patterns, but also agreement with the dynamics of the rupture growth.

The opening and propagation of a floral pore in a circular membrane patch can be observed in Figure 4a in an experiment, and Figure $4 \mathrm{~b}$ in a simulation where $g\left(=R_{c u r r}-R_{i}\right)$ represents the CA generation number of the snapshot. The key parameters in the simulation are $P_{\text {pin }}=2 \%$ and $P_{\text {cluster }}=0 \%$, meaning that all the pins in the model are of the dilute type and there are no pinned clusters. In experiments, the floral rupture is seen to advance along multiple fronts across membrane, which our CA is able to capture. We note the similarity of the boundary of the rupture front between the simulation and experiment. In general, increasing $P_{\text {pin }}$ (i.e., increasing the number of pin sites) would lead to smaller circular ruptures and a more varied rupture front boundary, whereas decreasing $P_{\text {pin }}$ would lead to larger circular ruptures and a more uniform rupture front boundary. Both cases have been observed experimentally.

Table 1 | Parameters used in the cellular automaton simulations shown in Fig. 4.

\begin{tabular}{|c|c|c|c|c|}
\hline & Meaning & Floral & Fractal & Mixed \\
\hline $\boldsymbol{P}_{\text {pin }}$ & Probability cell is pinned (dilute) & $2 \%$ & $0.4 \%$ & $4 \%$ \\
\hline $\boldsymbol{P}_{\text {cluster }}$ & Probability a pinned cell becomes root of a pin cluster (dense) & $0 \%$ & $100 \%$ & $0.08 \%$ \\
\hline $\boldsymbol{P}_{\text {perc }}$ & Probability cells adjacent to pin cluster cell become pinned & NA & *see Eq (6) & $36 \%$ \\
\hline $\boldsymbol{P}_{\text {offshoot }}$ & Probability a ruptured cluster generates a new pinned cluster & NA & $70 \%$ & $70 \%$ \\
\hline $\boldsymbol{T}_{\boldsymbol{a d h e s i o n}}$ & Tension due to lipid adhesion to surface & 0.2 & 0.2 & 0.2 \\
\hline $\boldsymbol{T}_{\text {reservoir }}$ & Tension due to MLV & 0.1 & 0.1 & 0.1 \\
\hline $\boldsymbol{T}_{\boldsymbol{c r i t}}$ & Critical tension threshold beyond which pinned cell ruptures & 0.8 & 0.75 & 0.8 \\
\hline $\boldsymbol{T}_{\boldsymbol{c l u s t e r}}$ & Additional cluster tension & Off & Off & On \\
\hline $\boldsymbol{C}$ & Pinned cell bond stiffness & 1 & 1 & 1 \\
\hline $\boldsymbol{R}_{\boldsymbol{i}}$ & Initial lipid radius & 100 & 100 & 100 \\
\hline $\boldsymbol{R}_{\boldsymbol{f}}$ & Final lipid radius & 150 & 150 & 150 \\
\hline$A P$ & Avalanche parameter & NA & 20 & 20 \\
\hline$C T P$ & Cluster tension parameter & NA & NA & 0.75 \\
\hline
\end{tabular}

Figure $4 c$ and $d$ shows examples of the purely fractal rupture morphology in an experiment and a simulation, respectively. The key difference in the simulation in this case versus the floral case is that we now set $P_{\text {cluster }}=100 \%$, meaning that all pins belong to a cluster and there are no dilute pins. In order to match the experiments in Figure $3 c$, in which ruptures begin toward the center, we turn $T_{\text {cluster }}$ off. In addition, we use a non-constant function for $P_{\text {perc }}$ 
$P_{\text {perc }}=\ln \left(\frac{R_{\text {curr }}}{2 R_{f}}+1\right)$

For the parameters used in this study, this leads to $0.288 \leq P_{\text {perc }} \leq 0.406$. The motivation for (6) is based in part on the observation of some experiments that clusters appear to become larger as the radius increases and also on one of the fundamental concepts of percolation theory-spanning clusters. Spanning clusters are those that fully extend across at least one dimension of a CA. Percolation models are marked by a phase transition at a critical threshold $P_{\text {perc }}^{\text {crit }}$, near and beyond which the chances of a spanning cluster appearing increases dramatically ${ }^{10}$. For a two-dimensional system utilizing Moore's neighborhood, such as the one we use in this study, $P_{\text {perc }}^{\text {crit }}$ is approximately $0.407^{10,31}$, which is just above the upper bound of (6). At $P_{\text {perc }}=P_{\text {perc }}^{\text {crit }}$, clusters are scale-invariant (i.e., are truly fractal in nature) ${ }^{10}$, and, in 2D, spanning clusters have a fractal dimension of $91 / 48^{32}$. In both simulation and experiment, we note that the ruptures begin towards the center of the membrane and grow into fractal structures that nearly reach the outer edge while trapping "islands" of lipid in the interior. We note that we use a slightly lower $T_{\text {crit }}$ in this case versus the other considered cases (see Table 1 ) in order to better match the experimental images. In general, we find that $T_{\text {crit }}$ between 0.7 and 0.8 matches experimental data well across the range of observed rupture morphologies.

Occasionally, some membrane patches display fractal and floral ruptures simultaneously. Figure 4e shows a typical experiment where the both types of ruptures are visible on the same patch. Figure $4 \mathrm{f}$ shows the snapshots from the corresponding CA simulation. We note that the ruptures in Figure $4 \mathrm{e}$ are primarily floral with only 2-3 identifiable clusters near the outer edge that are relatively small. Given the apparent dominance of dilute pinning, we use a very small cluster probability of $P_{\text {cluster }}=0.08 \%$. In addition, since the clusters are relatively small, we use a constant percolation probability sufficiently far from the critical threshold $\left(P_{\text {perc }}=36 \%\right)$. To account for the clusters initialing closer to the membrane edge, we use a non-zero $T_{\text {cluster }}$ with a $C T P=0.75$. Comparing experiments with simulation, we see that both initially rupture in a fractal cluster before eventually breaking into a floral pattern, which spreads throughout. 


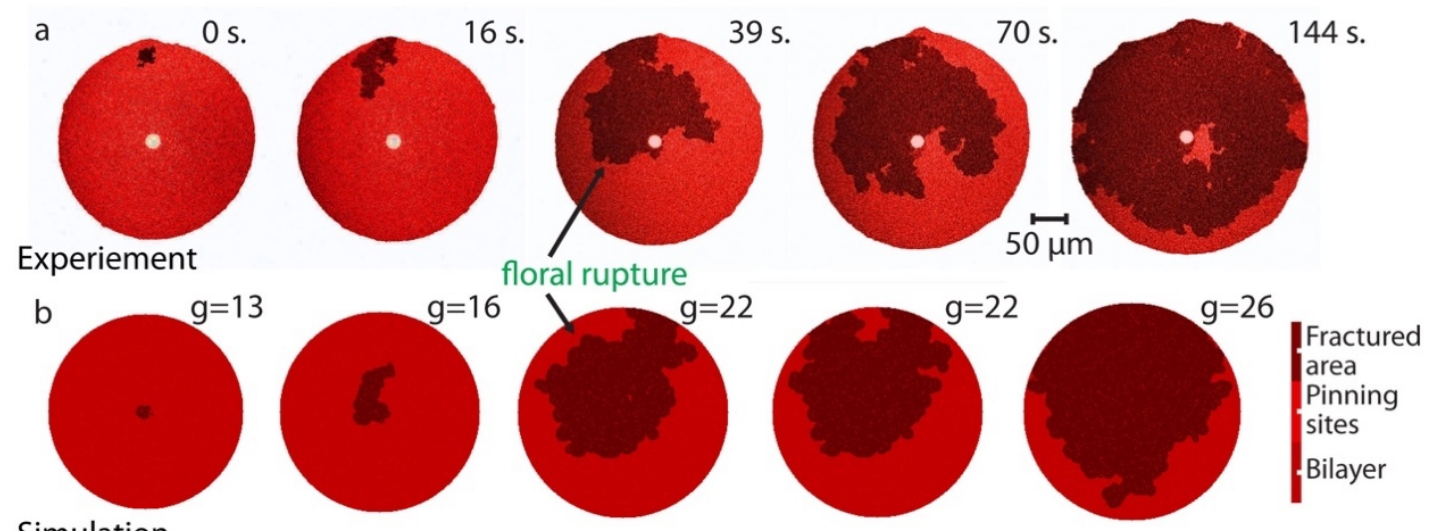

Simulation

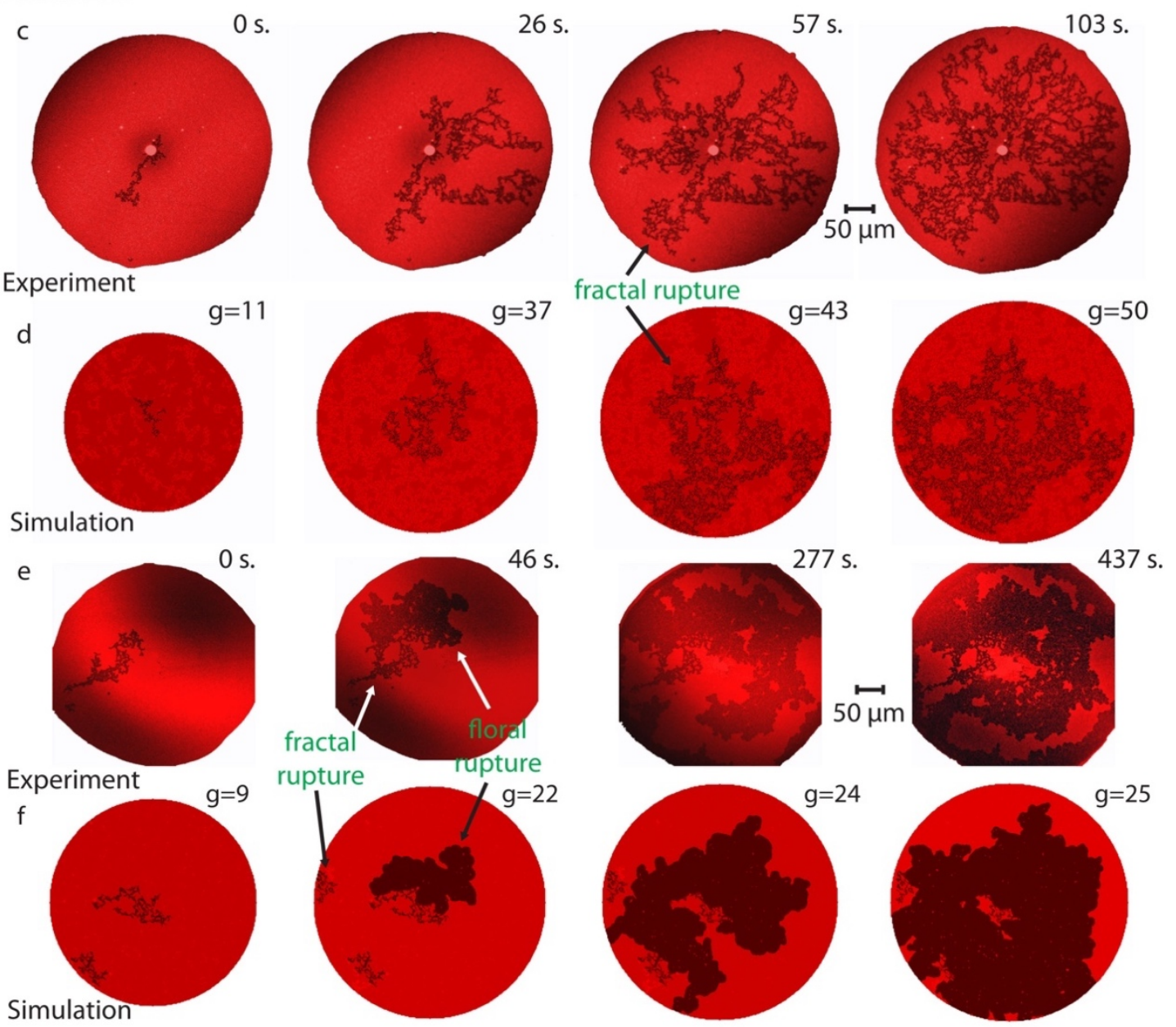

Figure 4 | Experiments and corresponding simulations. The opening and propagation of a floral pore in a circular membrane patch from top view in (a) an experiment and (b) simulation. The membrane in the experiments is doped with a fluorescence dye which makes it visible. In the simulations different colors represent from the most to less intense: the pinning sites, the bilayer (distal membrane), fractured area (the proximal membrane) and the background (surface). Fracturing of a circular membrane patch in (c) an experiment and (d) in simulations, from top view. The fractures appear in fractal morphology therefore are called the fractal ruptures. The membranes displaying the fractal and floral ruptures simultaneously (e) in an experiment and (f) in simulations. $g$ represents the CA generation number. 
In order to characterize the fractal rupture morphology, we calculate their fractal dimension using the box counting method ${ }^{33,34}$. In this approach, binary images of the rupture pattern are covered with sets of square boxes of successively decreasing size. The number of boxes including a piece of the rupture are recorded as a function box size in pixels. This relation, in logarithmic scale, yields an approximately linear plot, whose slope is the fractal dimension $D$. In Figure 5, we compare the results of the box counting method for experimental and simulated fractal ruptures shown in the second panels of Figure $4 \mathrm{c}$ and $\mathrm{d}$. We take close-up images of the ruptures (Fig. 5a and c) and convert them to binary images (Fig. 5b and d, respectively) where ruptured regions are assigned the value 1 and non-ruptured regions are assigned the value 0 . The box counting dimension can be approximated from the graphs shown in Figure $5 e$ by fitting a line to the data and computing the slope. This approach yields an approximate fractal dimension of $D_{\text {exp }} \approx 1.57$ for the experimental rupture and $D_{C A} \approx 1.65$ for the simulation $\left(P_{\text {perc }}=0.376\right)$. These values are close to each other, and in fact, we see generally good agreement between experiment and simulation in the box counting results across a range of box sizes (Fig. 5e). These results are also in line with those of the experiments and simulations in our previous work ${ }^{19}$. We note that, despite the noninteger dimension, these ruptures are not truly fractal in nature. The scaling behavior of lipid membrane is limited when approaching the molecular level, and the scaling behavior of the CA is limited by the finite number of cells used.
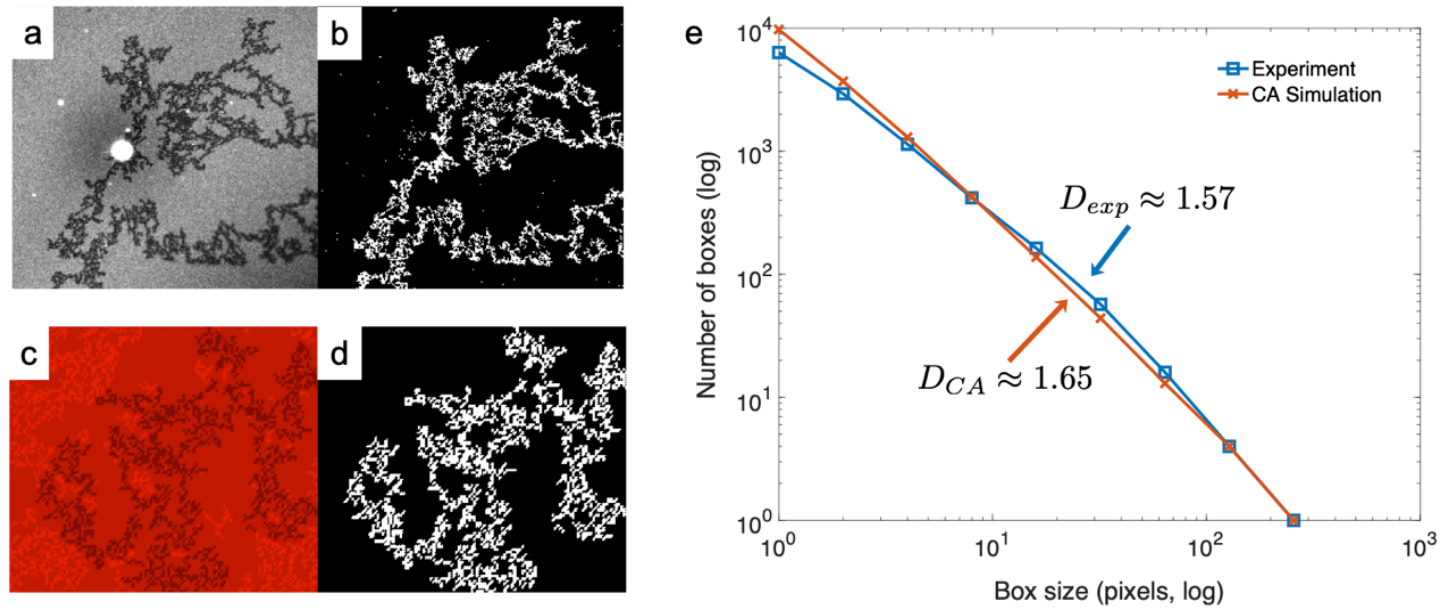

Figure 5| Fractal dimension comparison. Close-up snapshots of fractal rupturing in experiment (a) and simulation (c) corresponding to the second panels in Figure $4 \mathrm{c}$ and d, respectively. These snapshots are converted into binary images $(\mathbf{b}, \mathbf{d})$ for application of a box-counting algorithm to determine fractal dimension. (e) The results of the box counting algorithm showing the number of boxes including part of the rupture versus the size of the boxes covering the binary images. The slope of the curves yields the fractal dimension $D$. The fractal dimensions shown are computed via the slope of a linear fit of the data.

Besides the peculiar characteristic morphology of the fractal pores, the rupture dynamics also differ from the floral ruptures as well as from the most commonly observed circular pores in biomembranes: the fractal ruptures appear intermittently where, in between each occurrence, there is an eventless period. This waiting time corresponds to the time required for the membrane tension to build up again due to 
ongoing adhesion. When a pore opens, the membrane relaxes and, for the next rupture to appear, the membrane tension needs to increase again to reach to critical point of lysis. The CA model we have established can successfully anticipate this behavior through the reduced tension $T_{r}$ defined in (3) (Fig. $6 a-d)$. The reduced tension is the expression of membrane relaxation on new offshoot clusters and is governed by the avalanche parameter $A P$. As $A P$ is increased, the waiting time between fractal ruptures increases. This behavior in a typical simulation is shown for several $A P$ values in Figure $6 \mathrm{~d}$, where the percentage of fractured lipid cells is plotted against the CA generation. Jumps in these plots correspond to the breaking of a cluster. We see that the number of jumps tends to decrease and the number of generations between jumps tends to increase as $A P$ increases.

The saltatory behavior in fractal rupture growth is in stark contrast to the smooth growth observed in floral ruptures. Figure $6 \mathrm{~h}$ shows typical floral growth behavior in the CA for several values of $P_{\text {pin }}$. Rather than proceeding in starts and stops, floral growth is steady and approximately logarithmic in nature. As $P_{\text {pin }}$ increases, the number of pinning sites increases leading to more ruptures and an attendant increase in rupture area in a given generation. The plots of Figure 6 show good agreement with similar plots of both fractal avalanche and floral rupture behavior observed experimentally in previous studies ${ }^{1,3}$.
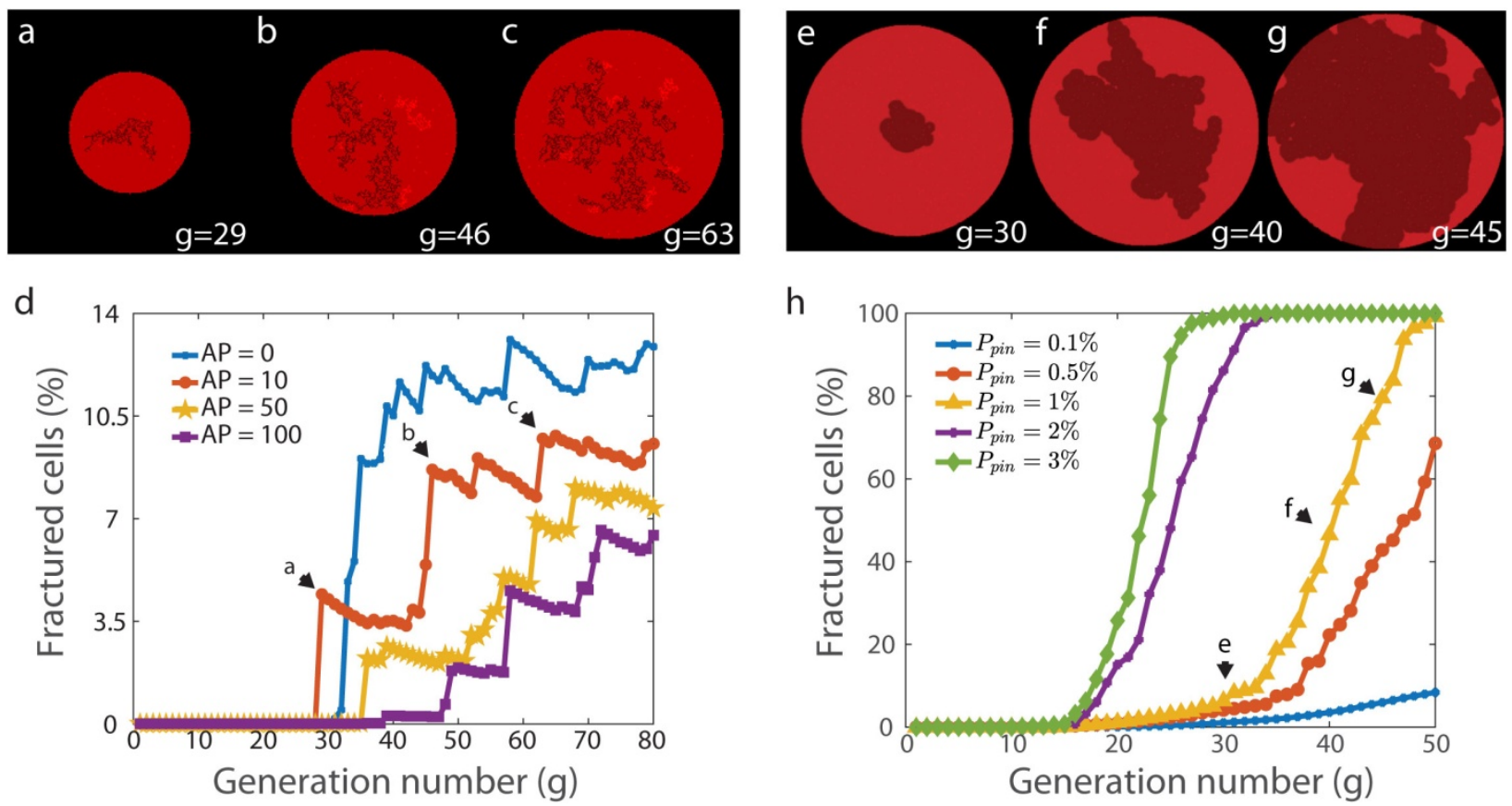

Figure 6| Simulated fractal and floral rupture dynamics. (a-c) Sequential CA snapshots depicting saltatory fractal rupturing corresponding to an avalanche parameter (AP) of 10. Fractured clusters form new offshoot pinned clusters, which eventually break and form additional cluster. (d) Plot showing the percentage of lipid cells fractured in the CA as a function of generation number for fractal ruptures, demonstrating the effect of the avalanche parameter on the intermittency of fractal rupture. Jumps correspond to a newly fractured cluster. (e-g) Sequential CA snapshots depicting floral rupturing corresponding to a $P_{\text {pin }}$ of $1 \%$. (h) Plot showing the percentage of lipid cells fractured in the CA as a function of generation number for floral ruptures for different (governed by $P_{\text {pin }}$ ). Growth in floral ruptures occurs smoothly, unlike in fractal avalanche fracture. 


\section{Conclusion}

In this study, we have proposed a novel cellular automaton that can successfully capture the varied fracture morphology and dynamics of lipid membrane rupture using a small number of parameters and simple rules. Dilute pins with circular ruptures lead to floral rupture morphologies, which spread continuously across the membrane. Cluster pins formed through a percolation process lead to fractal ruptures. Fractal ruptures are punctuated by periods of relaxation, which cause the ruptures to spread intermittently.

Cellular automata offer a couple advantages over spatio-temporal continuum models such as classical elasticity theory or peridynamics. First, they are based on a simple framework of rules and decisions, which make them more accessible to investigators from a broader range of backgrounds. In addition, they are significantly less computationally expensive. However, a CA is somewhat of an abstraction of the real lipid system, substituting "cells" for spatial area and "generations" for time. Its primary utility is in gaining insight into the pattern formation and avalanche dynamics and how it relates to pinning. We anticipate that the results of CA simulations can help inform the physics (e.g. constitutive relationships, tension, and pinning distribution) in a more-sophisticated continuum model. We anticipate a researcher modeling lipid growth and rupture could easily perform a parameter study to tune the parameters to their specific system and investigate a wide variety of combinations and their resulting rupture patterns. The model could also be expanded to allow for a user-defined pinning distribution, which would help in designing experiments aimed at controlling the pinning.

It is important to note that the non-trivial ruptures do not occur in extreme conditions but at moderate tensile stress, with similar values to those observed in living cells ${ }^{35,36}$. Such fractures are therefore anticipated to occur in vivo, although the findings are relatively new and currently very little is known regarding the formation of fractures in living organisms. An exception is the earlier observation of fractal pores reported in cultured Chinese Hamster Ovary cells' plasma membranes ${ }^{1}$. It is high likely that such ruptures continuously occur in tissue as a result of cell-to-cell adhesion or due to pinning of the plasma membrane of the cells to the underlying support: cytoskeleton ${ }^{37}$. Understanding the rules and, ultimately, the physics that underpin these morphologies can help researchers establish the governing factors for the formation of the membrane ruptures thus membrane damage and repair.

\section{Conflicts of interest}

There are no conflicts to declare.

\section{Acknowledgements}

IG acknowledges The Research Council of Norway (Forskningsrådet) Project Grant 274433, UiO: Life Sciences Convergence Environment, the Swedish Research Council (Vetenskapsrådet) Project Grant 201504561; start-up funding provided by the Centre for Molecular Medicine Norway \& Faculty of Mathematics and Natural Sciences at the University of Oslo. MT and AG acknowledge the support of the Kuehler Undergraduate Research Grant and start-up funding provided by the School of Engineering at Santa Clara University. The authors thank Ms. Ganna Reint for contributions to the experiments. 


\section{References}

1. I. Gözen, P. Dommersnes, I. Czolkos, A. Jesorka, T. Lobovkina and O. Orwar, Nature Materials, 2010, 9, 908.

2. K.-i. Akashi, H. Miyata, H. Itoh and K. Kinosita, Biophysical Journal, 1998, 74, 2973-2982.

3. O. Shindell, N. Mica, K. H. Cheng, E. Wang and V. D. Gordon, Langmuir, 2018, 34, 4673-4680.

4. I. Gozen, http://publications.lib.chalmers.se/records/fulltext/173294/173294.pdf, 2013.

5. H. K. Sarma, B. B. Maini and K. Jha, 1998, DOI: 10.2118/98-07-06.

6. B. Jha, L. Cueto-Felgueroso and R. Juanes, Physical Review Letters, 2011, 106, 194502.

7. F. R. Abbas, D. A., Earth Interactions, 2010, 14, 1-13.

8. W. Mather, O. Mondragón-Palomino, T. Danino, J. Hasty and L. S. Tsimring, Physical Review Letters, 2010, 104, 208101.

9. A. C. Callan-Jones, J. F. Joanny and J. Prost, Physical Review Letters, 2008, 100, 258106.

10. D. O'Sullivan and G. L. W. Perry, Spatial Simulation - Exploring Pattern and Process, WileyBlackwell, 2013.

11. A. Deutsch and S. Dormann, Cellular Automaton Modeling of Biological Pattern Formation

Characterization, Examples, and Analysis, Birkhäuser Boston, 2017.

12. A. W. Burks, Essays on Cellular Automata University of Illinois Press 1971.

13. J. v. Neumann, Theory of Self-Replicating Automata, University of Illinois Press, 1966.

14. M. Gardner, Scientific American, 1970, 223, 120-123.

15. S. Wolfram, Theory and Applications of Cellular Automata, World Scientific Pub Co Inc,, 1986.

16. S. A. Silling, Journal of the Mechanics and Physics of Solids, 2000, 48, 175-209.

17. E. O. Erdogan Madenci, Peridynamic Theory and its Applications, Springer, 2014.

18. S. A. Silling and E. Askari, Computers and Structures, 2005, 83, 1526-1535.

19. M. Taylor, I. Gözen, S. Patel, A. Jesorka and K. Bertoldi, PLOS ONE, 2016, 11, e0165947.

20. P. L. McNeil and R. A. Steinhardt, The Journal of Cell Biology, 1997, 137, 1.

21. S. Yasuda, D. Townsend, D. E. Michele, E. G. Favre, S. M. Day and J. M. Metzger, Nature, 2005, 436, 1025.

22. J. Hardy, The Lancet Neurology, 2017, 16, 342.

23. A. Schroeder, J. Kost and Y. Barenholz, Chemistry and Physics of Lipids, 2009, 162, 1-16.

24. J.-M. Escoffre, T. Portet, L. Wasungu, J. Teissié, D. Dean and M.-P. Rols, Molecular Biotechnology, 2009, 41, 286-295.

25. A. Ainla, I. Gözen, O. Orwar and A. Jesorka, Analytical Chemistry, 2009, 81, 5549-5556.

26. T. Lobovkina, I. Gözen, Y. Erkan, J. Olofsson, S. G. Weber and O. Orwar, Soft Matter, 2010, 6, 268-272.

27. T. Harayama and H. Riezman, Nature Reviews Molecular Cell Biology, 2018, 19, 281-296.

28. I. Gozen, P. Dommersnes, O. Orwar and A. Jesorka, Soft Matter, 2012, 8, 6220-6225.

29. Y. Roiter, M. Ornatska, A. R. Rammohan, J. Balakrishnan, D. R. Heine and S. Minko, Nano Letters, 2008, 8, 941-944.

30. I. Gözen, B. Ortmen, I. Põldsalu, P. Dommersnes, O. Orwar and A. Jesorka, Soft Matter, 2013, 9, 2787-2792.

31. K. Malarz and S. Galam, Physical Review E, 2005, 71, 016125.

32. S. Havlin and D. ben-Avraham, Diffusion and Reactions in Fractals and Disordered Systems (Cambridge Nonlinear Science Series), Cambridge University Press, 2000.

33. K. Falconer, Fractal Geometry: Mathematical Foundations and Applications, Wiley, 3rd edn., 2014. 
34. T. G. Smith Jr, G. D. Lange and W. B. Marks, Journal of Neuroscience Methods, 1996, 69, 123136.

35. P. L. McNeil and T. Kirchhausen, Nature Reviews Molecular Cell Biology, 2005, 6, 499-505.

36. I. Gozen and P. Dommersnes, European Physical Journal: Special Topics, 2014, 223, 1813-1829.

37. C. G. Zervas, S. L. Gregory and N. H. Brown, Journal of Cell Biology, 2001, 152, 1007-1018. 